\title{
Half convex functions
}

\section{Zlatko Pavić*}

${ }^{*}$ Correspondence: Zlatko.Pavic@sfsb.hr

Mechanical Engineering Faculty in Slavonski Brod, University of Osijek, Trg Ivane Brlić Mažuranić 2,

Slavonski Brod, 35000, Croatia

\begin{abstract}
The paper provides the characteristic properties of half convex functions. The analytic and geometric image of half convex functions is presented using convex combinations and support lines. The results relating to convex combinations are applied to quasi-arithmetic means.
\end{abstract}

MSC: $26 \mathrm{~A} 51 ; 26 \mathrm{D} 15$

Keywords: convex combination center; half convex function; Jensen's inequality; quasi-arithmetic mean

\section{Introduction}

Through the paper we will use an interval $\mathcal{I} \subseteq \mathbb{R}$ with the non-empty interior $\mathcal{I}^{0}$. Two subintervals of $\mathcal{I}$ specified by the point $c \in \mathcal{I}^{0}$ will be denoted by

$$
\mathcal{I}_{x \geq c}=\{x \in \mathcal{I}: x \geq c\} \quad \text { and } \quad \mathcal{I}_{x \leq c}=\{x \in \mathcal{I}: x \leq c\} .
$$

We say that a function $f: \mathcal{I} \rightarrow \mathbb{R}$ is right convex if it is convex on $\mathcal{I}_{x \geq c}$ for some point $c \in \mathcal{I}^{0}$. A left convex function is similarly defined on the interval $\mathcal{I}_{x \leq c}$. A function is half convex if it is right or left convex.

A combination

$$
c=\sum_{i=1}^{n} p_{i} x_{i}
$$

of points $x_{i}$ and real coefficients $p_{i}$ is affine if the coefficient sum $\sum_{i=1}^{n} p_{i}=1$. The above combination is convex if all coefficients $p_{i}$ are non-negative and the coefficient sum is equal to 1 . The point $c$ itself is called the combination center, and it is important for mathematical inequalities.

Every affine function $f: \mathbb{R} \rightarrow \mathbb{R}$ satisfies the equality

$$
f\left(\sum_{i=1}^{n} p_{i} x_{i}\right)=\sum_{i=1}^{n} p_{i} f\left(x_{i}\right)
$$

for all affine combinations from $\mathbb{R}$. Every convex function $f: \mathcal{I} \rightarrow \mathbb{R}$ satisfies the Jensen inequality

$$
f\left(\sum_{i=1}^{n} p_{i} x_{i}\right) \leq \sum_{i=1}^{n} p_{i} f\left(x_{i}\right)
$$

for all convex combinations from $\mathcal{I}$.

\section{照 Springer}

(2014 Pavić; licensee Springer. This is an Open Access article distributed under the terms of the Creative Commons Attribution License (http://creativecommons.org/licenses/by/2.0), which permits unrestricted use, distribution, and reproduction in any medium, provided the original work is properly cited. 
If $a, b \in \mathbb{R}$ are different numbers, say $a<b$, then every number $x \in \mathbb{R}$ can be uniquely presented as the affine combination

$$
x=\frac{b-x}{b-a} a+\frac{x-a}{b-a} b .
$$

The above binomial combination of $a$ and $b$ is convex if, and only if, the number $x$ belongs to the interval $[a, b]$. Given the function $f: \mathbb{R} \rightarrow \mathbb{R}$, let $f_{\{a, b\}}^{\text {line }}: \mathbb{R} \rightarrow \mathbb{R}$ be the function of the line passing through the points $(a, f(a))$ and $(b, f(b))$ of the graph of $f$. Using the affinity of $f_{\{a, b\}}^{\text {line }}$, we get the equation

$$
f_{\{a, b\}}^{\operatorname{line}}(x)=\frac{b-x}{b-a} f(a)+\frac{x-a}{b-a} f(b) .
$$

Assuming that $f$ is convex, we get the inequality

$$
f(x) \leq f_{\{a, b\}}^{\text {line }}(x) \quad \text { if } x \in[a, b]
$$

and the reverse inequality

$$
f(x) \geq f_{\{a, b\}}^{\text {line }}(x) \quad \text { if } x \notin(a, b) .
$$

A general overview of convexity, convex functions, and applications can be found in [1], and many details of this branch are in [2]. The different forms of the famous Jensen's inequality (discrete form in [3] and integral form in [4]) can be found in [5] and [6]. Global bounds for Jensen's functional were investigated in [7].

\section{Recent results}

The following results on half convex functions have been presented in [8].

WRCF-Theorem (Weighted right convex function theorem) Let $f(x)$ be a function defined on $\mathcal{I}$ and convex for $x \geq c \in \mathcal{I}$, and let $p_{1}, \ldots, p_{n}$ be positive real numbers such that

$$
p=\min \left\{p_{1}, \ldots, p_{n}\right\}, \quad p_{1}+\cdots+p_{n}=1 .
$$

The inequality

$$
f\left(\sum_{i=1}^{n} p_{i} x_{i}\right) \leq \sum_{i=1}^{n} p_{i} f\left(x_{i}\right)
$$

holds for all $x_{1}, \ldots, x_{n} \in \mathcal{I}$ satisfying $\sum_{i=1}^{n} p_{i} x_{i} \geq c$ if and only if the inequality

$$
f(p x+(1-p) y) \leq p f(x)+(1-p) f(y)
$$

holds for all $x, y \in \mathcal{I}$ such that $x \leq c \leq y$ and $p x+(1-p) y=c$.

The WLCF-Theorem (weighted left convex function theorem) is presented in a similar way. The final common theorem on half convexity reads as follows. 
WHCF-Theorem (Weighted half convex function theorem) Let $f(x)$ be a function defined on $\mathcal{I}$ and convex for $x \geq c$ or $x \leq c$, where $c \in \mathcal{I}$, and let $p_{1}, \ldots, p_{n}$ be positive real numbers such that

$$
p=\min \left\{p_{1}, \ldots, p_{n}\right\}, \quad p_{1}+\cdots+p_{n}=1 .
$$

The inequality

$$
f\left(\sum_{i=1}^{n} p_{i} x_{i}\right) \leq \sum_{i=1}^{n} p_{i} f\left(x_{i}\right)
$$

holds for all $x_{1}, \ldots, x_{n} \in \mathcal{I}$ satisfying $\sum_{i=1}^{n} p_{i} x_{i}=c$ if and only if the inequality

$$
f(p x+(1-p) y) \leq p f(x)+(1-p) f(y)
$$

holds for all $x, y \in \mathcal{I}$ such that $p x+(1-p) y=c$.

\section{Main results}

\subsection{Half convexity with convex combinations}

The main result is Lemma 3.1, which extends the right convexity of WRCF-Theorem to all convex combinations, without pre-selected coefficients $p_{1}, \ldots, p_{n}$ and without emphasizing the coefficient $p=\min \left\{p_{1}, \ldots, p_{n}\right\}$. Therefore, Jensen's inequality for right convex functions follows (using as usual non-negative coefficients in convex combinations).

Lemma 3.1 Let $f: \mathcal{I} \rightarrow \mathbb{R}$ be a function that is convex on $\mathcal{I}_{x \geq c}$ for some point $c \in \mathcal{I}^{0}$. Then the inequality

$$
f\left(\sum_{i=1}^{n} p_{i} x_{i}\right) \leq \sum_{i=1}^{n} p_{i} f\left(x_{i}\right)
$$

holds for all convex combinations from $\mathcal{I}$ satisfying $\sum_{i=1}^{n} p_{i} x_{i} \geq c$ if, and only if, the inequality

$$
f(p x+q y) \leq p f(x)+q f(y)
$$

holds for all binomial convex combinations from $\mathcal{I}$ satisfying $p x+q y=c$.

Proof Let us prove the sufficiency using the induction on the integer $n \geq 2$.

The base of induction. Take a binomial convex combination from $\mathcal{I}$ satisfying $p x+q y \geq c$ with $x \leq y$ and $0<p<1$. If $x \geq c$, we apply the Jensen inequality to get the inequality in equation (3.1) for $n=2$. If $x<c$, we have $x<c \leq p x+q y<y$. Since $p x+q y$ lies between $c$ and $y$, we have some convex combination $p_{0} c+q_{0} y=p x+q y$ with $p_{0}>0$. Then the affine combination

$$
c=\frac{p}{p_{0}} x+\frac{q-q_{0}}{p_{0}} y
$$


is convex since $c$ lies between $x$ and $y$, and hence

$$
f(c) \leq \frac{p}{p_{0}} f(x)+\frac{q-q_{0}}{p_{0}} f(y)
$$

by assumption in equation (3.2). Using the convexity of $f$ on $\mathcal{I}_{x \geq c}$ and the above inequality, it follows that

$$
f(p x+q y)=f\left(p_{0} c+q_{0} y\right) \leq p_{0} f(c)+q_{0} f(y) \leq p f(x)+q f(y) .
$$

The step of induction. Suppose that the inequality in equation (3.1) is true for all corresponding $n$-membered convex combinations with $n \geq 2$. Take an $(n+1)$-membered convex combination from $\mathcal{I}$ satisfying $\sum_{i=1}^{n+1} p_{i} x_{i} \geq c$ with $x_{1}=\min \left\{x_{1}, \ldots, x_{n}\right\}$ and $0<p_{1}<1$. If $x_{1} \geq c$, we can use the Jensen inequality to get the inequality in equation (3.1) for $n+1$. If $x_{1}<c$, relying on the representation

$$
\sum_{i=1}^{n+1} p_{i} x_{i}=p_{1} x_{1}+\left(1-p_{1}\right) \sum_{i=2}^{n+1} \frac{p_{i}}{1-p_{1}} x_{i}
$$

we can apply the induction base with the points $x=x_{1}$ and $y=\sum_{i=2}^{n+1} p_{i} /\left(1-p_{1}\right) x_{i}$, and the coefficients $p=p_{1}$ and $q=1-p_{1}$. Using the inequality in equation (3.5) and the induction premise

$$
f(y)=f\left(\sum_{i=2}^{n+1} \frac{p_{i}}{1-p_{1}} x_{i}\right) \leq \sum_{i=2}^{n+1} \frac{p_{i}}{1-p_{1}} f\left(x_{i}\right),
$$

we get the inequality in equation (3.1) for the observed $(n+1)$-membered combination.

Remark 3.2 The formula in equation (3.5) for the case $x<c$ in the proof of Lemma 3.1 can be derived with exactly calculated convex combination coefficients. If $d=p x+q y$, then we have the order $x<c \leq d<y$. Using the presentation formula in equation (1.4), we determine the convex combinations

$$
d=\frac{y-d}{y-c} c+\frac{d-c}{y-c} y, \quad c=\frac{y-c}{y-x} x+\frac{c-x}{y-x} y .
$$

Applying the right convex function $f$, it follows that

$$
\begin{aligned}
f(p x+q y) & =f(d) \leq \frac{y-d}{y-c} f(c)+\frac{d-c}{y-c} f(y) \\
& \leq \frac{y-d}{y-c}\left[\frac{y-c}{y-x} f(x)+\frac{c-x}{y-x} f(y)\right]+\frac{d-c}{y-c} f(y) \\
& =\frac{y-d}{y-x} f(x)+\frac{d-x}{y-x} f(y)=p f(x)+q f(y)
\end{aligned}
$$

since

$$
d=\frac{y-d}{y-x} x+\frac{d-x}{y-x} y .
$$


Following Lemma 3.1 we can state a similar lemma for left convex functions. So, if a function $f(x)$ is convex on $\mathcal{I}_{x \geq c}$, and if the interval $\mathcal{I}$ is symmetric respecting the point $c$, then the function $g(x)=f(2 c-x)$ is convex on $\mathcal{I}_{x \leq c}$. The function $g$ verifies the same inequalities as the function $f$ in Lemma 3.1, wherein the condition $\sum_{i=1}^{n} p_{i}\left(2 c-x_{i}\right) \geq c$ becomes $\sum_{i=1}^{n} p_{i} x_{i} \leq c$.

Connecting the lemmas on right and left convex functions, we have the following result for half convex functions.

Theorem 3.3 Let $f: \mathcal{I} \rightarrow \mathbb{R}$ be a function that is convex on $\mathcal{I}_{x \geq c}$ or $\mathcal{I}_{x \leq c}$ for some point $c \in \mathcal{I}^{0}$. Then the inequality

$$
f\left(\sum_{i=1}^{n} p_{i} x_{i}\right) \leq \sum_{i=1}^{n} p_{i} f\left(x_{i}\right)
$$

holds for all convex combinations from $\mathcal{I}$ satisfying $\sum_{i=1}^{n} p_{i} x_{i}=c$ if, and only if, the inequality

$$
f(p x+q y) \leq p f(x)+q f(y)
$$

holds for all binomial convex combinations from $\mathcal{I}$ satisfying $p x+q y=c$.

Iff is concave on $\mathcal{I}_{x \geq c}$ or $\mathcal{I}_{x \leq c}$ for some point $c \in \mathcal{I}^{0}$, then the reverse inequalities are valid in equations (3.8) and (3.9).

\subsection{Half convexity with support lines}

The main result is Lemma 3.5 complementing a geometric image of a right convex function satisfying Jensen's inequality for all binomial convex combinations with the center at the convexity edge-point $c$. The graph of such a function is located above the right tangent line at $c$.

Regardless of convexity, we start with a trivial lemma which can be taken as Jensen's inequality for functions supported with the line passing through a point of its graph.

Lemma 3.4 Let $f: \mathcal{I} \rightarrow \mathbb{R}$ be a function, and let $C(c, f(c))$ be a point of the graph off with $c \in \mathcal{I}^{0}$.

If there exists a line that passes through the point $C$ below the graph off, then the function $f$ satisfies the inequality

$$
f\left(\sum_{i=1}^{n} p_{i} x_{i}\right) \leq \sum_{i=1}^{n} p_{i} f\left(x_{i}\right)
$$

for all convex combinations from $\mathcal{I}$ satisfying $\sum_{i=1}^{n} p_{i} x_{i}=c$.

If there exists a line that passes through the point $C$ above the graph off, then the reverse inequality is valid in equation (3.10).

Proof Let $f_{\{c\}}^{\text {line }}$ be the function of the line passing through the point $C$. Assume the case $f_{\{c\}}^{\text {line }} \leq f$. If $\sum_{i=1}^{n} p_{i} x_{i}=c$ is any convex combination from $\mathcal{I}$ with the center at $c$, then using 


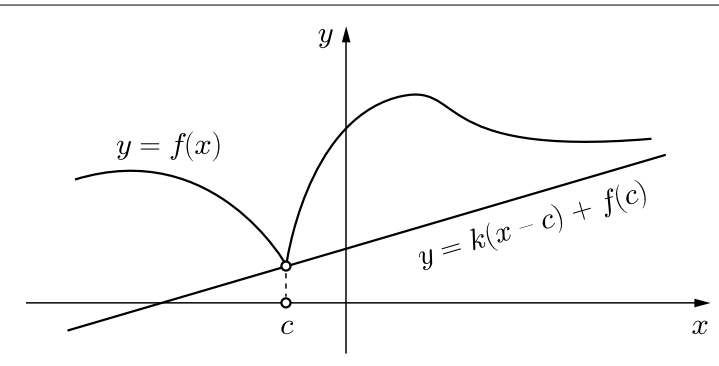

Figure 1 Function supported by the line.

the affinity of $f_{\{c\}}^{\text {line }}$, it follows that

$$
\begin{aligned}
f\left(\sum_{i=1}^{n} p_{i} x_{i}\right) & =f_{\{c\}}^{\text {line }}\left(\sum_{i=1}^{n} p_{i} x_{i}\right)=\sum_{i=1}^{n} p_{i} f_{\{c\}}^{\text {line }}\left(x_{i}\right) \\
& \leq \sum_{i=1}^{n} p_{i} f\left(x_{i}\right),
\end{aligned}
$$

concluding the proof in the case that the line is below the curve.

The function $f$ with the line passing through the point $C$ below its graph is shown in Figure 1. In what follows, $f$ will be convex to the right or left of $c$, and the right or left tangent line will be used as the support line at $c$.

If $f$ is convex on $\mathcal{I}_{x \geq c}$, then the slope $(f(x)-f(c)) /(x-c)$ approaches the real number $k_{r}=f^{\prime}(c+)$ or to the negative infinity $-\infty$ as $x$ approaches $c+$.

Lemma 3.5 Let $f: \mathcal{I} \rightarrow \mathbb{R}$ be a function that is convex on $\mathcal{I}_{x \geq c}$ for some point $c \in \mathcal{I}^{0}$. Let $k_{r} \in \mathbb{R}$ be the slope of the right tangent line of the function $f$ at the point $c$.

Then the inequality

$$
f(p x+q y) \leq p f(x)+q f(y)
$$

holds for all binomial convex combinations from $\mathcal{I}$ satisfying $p x+q y=c$ if, and only if, the right tangent line inequality

$$
f(x) \geq k_{r}(x-c)+f(c)
$$

holds for all points $x \in \mathcal{I}$.

Proof The proof of necessity. Since the tangent line of a convex function is the support line, it is sufficient to prove the inequality in equation (3.12) for all $x$ in $\mathcal{I}$ which are less than $c$. Take any such $x$. Then the $c$-centered convex combinations $p x+q y=c$ from $\mathcal{I}$ include only $y>c$. The inequality in equation (3.11) with these combinations can be adapted to the form

$$
\frac{f(x)-f(c)}{x-c} \leq \frac{f(y)-f(c)}{y-c} .
$$




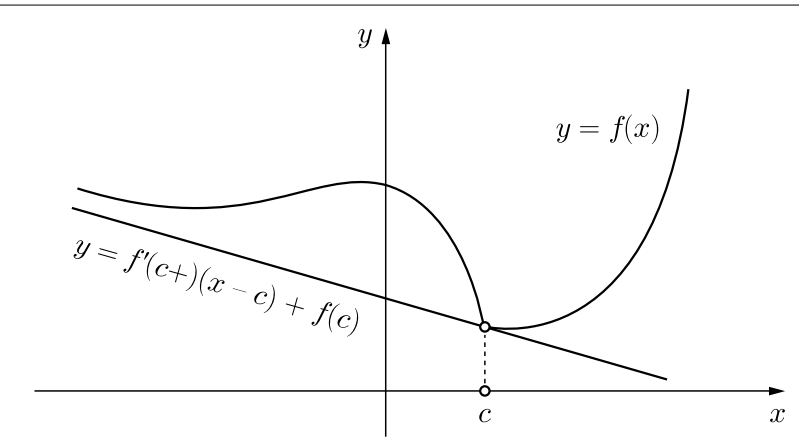

Figure 2 Right convex function supported by the right tangent line.

Letting $y$ go to $c$ and $p$ to 0 so that $x$ stays the same, it follows that

$$
\frac{f(x)-f(c)}{x-c} \leq \lim _{y \rightarrow c+} \frac{f(y)-f(c)}{y-c}=f^{\prime}(c+)=k_{r} .
$$

Multiplying the above inequality with $x-c<0$, we get the inequality in equation (3.12).

The proof of sufficiency. Using the convex function $g: \mathcal{I} \rightarrow \mathbb{R}$ defined by

$$
g(x)= \begin{cases}k_{r}(x-c)+f(c) & \text { for } x \leq c \\ f(x) & \text { for } x \geq c\end{cases}
$$

we see that the inequality

$$
f(p x+q y)=g(p x+q y) \leq p g(x)+q g(y) \leq p f(x)+q f(y)
$$

holds for all binomial convex combinations from $\mathcal{I}$ satisfying $p x+q y=c$.

The graph of the right convex function $f$ with the right tangent line at the point $c$ is presented in Figure 2.

Based on Lemma 3.5 and its analogy for left convex functions, and also on Theorem 3.3, we have the following characterization of half convex and half concave functions.

Theorem 3.6 Let $f: \mathcal{I} \rightarrow \mathbb{R}$ be a function that is convex on $\mathcal{I}_{x \geq c}$ or $\mathcal{I}_{x \leq c}$ for some point $c \in \mathcal{I}^{0}$. Iff is right (resp. left) convex, let $k_{r} \in \mathbb{R}$ (resp. $\left.k_{l}\right)$ be the slope of the right (resp. left) tangent line at $c$.

Then the following three conditions are equivalent:

(1) The inequality

$$
f\left(\sum_{i=1}^{n} p_{i} x_{i}\right) \leq \sum_{i=1}^{n} p_{i} f\left(x_{i}\right)
$$

holds for all convex combinations from $\mathcal{I}$ satisfying $\sum_{i=1}^{n} p_{i} x_{i}=c$.

(2) The inequality

$$
f(p x+q y) \leq p f(x)+q f(y)
$$

holds for all binomial convex combinations from $\mathcal{I}$ satisfying $p x+q y=c$. 
(3) The tangent line inequality

$$
f(x) \geq k(x-c)+f(c)
$$

with $k=k_{r}$ or $k=k_{l}$ holds for all points $x \in \mathcal{I}$.

Iff is concave on $\mathcal{I}_{x \geq c}$ or $\mathcal{I}_{x \leq c}$ for some point $c \in \mathcal{I}^{0}$, then the reverse inequalities are valid in (1), (2), and (3).

\section{Application to quasi-arithmetic means}

In the applications of convexity to quasi-arithmetic means, we use strictly monotone continuous functions $\varphi, \psi: \mathcal{I} \rightarrow \mathbb{R}$ such that $\psi$ is $\varphi$-convex, that is, the function $f=\psi \circ \varphi^{-1}$ is convex (according to the terminology in [2, Definition 1.19]). A similar notation is used for concavity.

A concept of quasi-arithmetic mean refers to convex combinations and strictly monotone continuous functions. The $\varphi$-quasi-arithmetic mean of the convex combination $\sum_{i=1}^{n} p_{i} x_{i}$ from $\mathcal{I}$ is the point

$$
M_{\varphi}\left(x_{i} ; p_{i}\right)=\varphi^{-1}\left(\sum_{i=1}^{n} p_{i} \varphi\left(x_{i}\right)\right)
$$

which belongs to $\mathcal{I}$, because the convex combination $\sum_{i=1}^{n} p_{i} \varphi\left(x_{i}\right)$ belongs to $\varphi(\mathcal{I})$. If $\varphi$ is the identity function on $\mathcal{I}$, then its quasi-arithmetic mean is just the convex combination $\sum_{i=1}^{n} p_{i} x_{i}$.

Right convexity and concavity for quasi-arithmetic means can be obtained using Lemma 3.1.

Corollary 4.1 Let $\varphi, \psi: \mathcal{I} \rightarrow \mathbb{R}$ be strictly monotone continuous functions, and $\mathcal{J}=\varphi(\mathcal{I})$ be the $\varphi$-image of the interval $\mathcal{I}$.

If $\psi$ is either $\varphi$-convex on $\mathcal{J}_{z \geq d}$ for some point $d=\varphi(c) \in \mathcal{J}^{0}$ and increasing on $\mathcal{I}$ or $\varphi$-concave on $\mathcal{J}_{z \geq d}$ and decreasing on $\mathcal{I}$, then the inequality

$$
M_{\varphi}\left(x_{i} ; p_{i}\right) \leq M_{\psi}\left(x_{i} ; p_{i}\right)
$$

holds for all convex combinations from $\mathcal{J}$ satisfying $\sum_{i=1}^{n} p_{i} \varphi\left(x_{i}\right) \geq d$ if, and only if, the inequality

$$
M_{\varphi}(x, y ; p, q) \leq M_{\psi}(x, y ; p, q)
$$

holds for all binomial convex combinations from $\mathcal{J}$ satisfying $p \varphi(x)+q \varphi(y)=d$.

If $\psi$ is either $\varphi$-convex on $\mathcal{J}_{z \geq \varphi(c)}$ for some point $d=\varphi(c) \in \mathcal{J}^{0}$ and decreasing on $\mathcal{I}$ or $\varphi$-concave on $\mathcal{J}_{z \geq d}$ and increasing on $\mathcal{I}$, then the reverse inequalities are valid in equations (4.2) and (4.3).

Proof We prove the case that the function $\psi$ is $\varphi$-convex on the interval $\mathcal{J}_{z \geq d}$ and increasing on the interval $\mathcal{I}$. Put $f=\psi \circ \varphi^{-1}$. 
In the first step, applying Lemma 3.1 to the function $f: \mathcal{J} \rightarrow \mathbb{R}$, convex on the interval $\mathcal{J}_{z \geq d}$, we get the equivalence: the inequality

$$
f\left(\sum_{i=1}^{n} p_{i} \varphi\left(x_{i}\right)\right) \leq \sum_{i=1}^{n} p_{i} f\left(\varphi\left(x_{i}\right)\right)
$$

holds for all convex combinations from $\mathcal{J}$ satisfying $\sum_{i=1}^{n} p_{i} \varphi\left(x_{i}\right) \geq d$ if, and only if, the inequality

$$
f(p \varphi(x)+q \varphi(y)) \leq p f(\varphi(x))+q f(\varphi(y))
$$

holds for all binomial convex combinations from $\mathcal{J}$ satisfying $p \varphi(x)+q \varphi(y)=d$.

In the second step, assigning the increasing function $\psi^{-1}$ to the above inequalities, the equivalence follows. The inequality

$$
M_{\varphi}\left(x_{i} ; p_{i}\right)=\varphi^{-1}\left(\sum_{i=1}^{n} p_{i} \varphi\left(x_{i}\right)\right) \leq \psi^{-1}\left(\sum_{i=1}^{n} p_{i} \psi\left(x_{i}\right)\right)=M_{\psi}\left(x_{i} ; p_{i}\right)
$$

holds for all convex combinations from $\mathcal{J}$ satisfying $\sum_{i=1}^{n} p_{i} \varphi\left(x_{i}\right) \geq d$ if, and only if, the inequality

$$
M_{\varphi}(x, y ; p, q)=\varphi^{-1}(p \varphi(x)+q \varphi(y)) \leq \psi^{-1}(p \varphi(x)+q \varphi(y))=M_{\psi}(x, y ; p, q)
$$

holds for all binomial convex combinations from $\mathcal{J}$ satisfying $p \varphi(x)+q \varphi(y)=d$.

Combining 'right' and 'left' similarly as in Theorem 3.3, we get the following characterization of half convexity and concavity for quasi-arithmetic means.

Corollary 4.2 Let $\varphi, \psi: \mathcal{I} \rightarrow \mathbb{R}$ be strictly monotone continuous functions, and let $\mathcal{J}=$ $\varphi(\mathcal{I})$ be the $\varphi$-image of the interval $\mathcal{I}$.

If $\psi$ is either $\varphi$-convex on $\mathcal{J}_{z \geq d}$ or $\mathcal{J}_{z \leq d}$ for some point $d=\varphi(c) \in \mathcal{J}^{0}$ and increasing on $\mathcal{I}$ or $\varphi$-concave on $\mathcal{J}_{z \geq d}$ or $\mathcal{J}_{z \leq d}$ and decreasing on $\mathcal{I}$, then the inequality

$$
M_{\varphi}\left(x_{i} ; p_{i}\right) \leq M_{\psi}\left(x_{i} ; p_{i}\right)
$$

holds for all convex combinations from $\mathcal{J}$ satisfying $\sum_{i=1}^{n} p_{i} \varphi\left(x_{i}\right)=d$ if, and only if, the inequality

$$
M_{\varphi}(x, y ; p, q) \leq M_{\psi}(x, y ; p, q)
$$

holds for all binomial convex combinations from $\mathcal{J}$ satisfying $p \varphi(x)+q \varphi(y)=d$.

If $\psi$ is either $\varphi$-convex on $\mathcal{J}_{z \geq \varphi(c)}$ or $\mathcal{J}_{z \leq d}$ for some point $d=\varphi(c) \in \mathcal{J}^{0}$ and decreasing on $\mathcal{I}$ or $\varphi$-concave on $\mathcal{J}_{z \geq d}$ or $\mathcal{J}_{z \leq d}$ and increasing on $\mathcal{I}$, then the reverse inequalities are valid in equations (4.4) and (4.5).

For a basic study of means and their inequalities the excellent book in [9] is recommended. Very general forms of discrete and integral quasi-arithmetic means and their 
refinements were studied in [6]. A simple transformation for deriving new means was introduced in [10].

In further research of half convexity it would be useful to include the functions of several variables. Their geometric characterization using support surfaces would be especially interesting.

\section{Competing interests}

The author declares that they have no competing interests.

Received: 5 September 2013 Accepted: 17 December 2013 Published: 09 Jan 2014

\section{References}

1. Niculescu, CP, Persson, LE: Convex Functions and Their Applications. CMS Books in Mathematics. Springer, New York (2006)

2. Pečarić, JE, Proschan, F, Tong, YL: Convex Functions, Partial Orderings, and Statistical Applications. Academic Press, New York (1992)

3. Jensen, JLWV: Om konvekse Funktioner og Uligheder mellem Middelværdier. Nyt Tidss. Mat. B 16, 49-68 (1905)

4. Jensen, JLWV: Sur les fonctions convexes et les inégalités entre les valeurs moyennes. Acta Math. 30, 175-193 (1906)

5. Pavić, Z, Pečarić, J, Perić, I: Integral, discrete and functional variants of Jensen's inequality. J. Math. Inequal. 5, 253-264 (2011)

6. Mićić, J, Pavić, Z, Pečarić, J: The inequalities for quasiarithmetic means. Abstr. Appl. Anal. 2012, Article ID 203145 (2012)

7. Sándor, J: On global bounds for generalized Jensen's inequality. Ann. Funct. Anal. 4, 18-24 (2013)

8. Cirtoaje, V, Baiesu, A: An extension of Jensen's discrete inequality to half convex functions. J. Inequal. Appl. 2011, Article ID 101 (2011)

9. Bullen, PS, Mitrinović, DS, Vasić, PM: Means and Their Inequalities. Reidel, Dordrecht (1988)

10. Raïssouli, M, Sándor, J: On a method of construction of new means with applications. J. Inequal. Appl. 2013, Article ID 89 (2013)

\section{Submit your manuscript to a SpringerOpen ${ }^{\circ}$ journal and benefit from:}

- Convenient online submission

Rigorous peer review

- Immediate publication on acceptance

- Open access: articles freely available online

- High visibility within the field

- Retaining the copyright to your article 